\title{
A non quasi exact solvable eigenvalue problem with PT-invariant non-Hermitian complex potential
}

\author{
Marwan Izzat El-Kawni, Abdulla Jameel Sous \\ College of Science, Al-Quds Open University, Nablus, PO Box 893, Palestine
}

Email address:

mkawni@qou.edu (M. I. El-Kawni), asous@qou.edu (A. J. Sous)

To cite this article:

Marwan Izzat El-Kawni, Abdulla Jameel Sous. A Non Quasi Exact Solvable Eigenvalue Problem with PT-Invariant Non-Hermitian Complex Potential. American Journal of Modern Physics. Vol. 4, No. 1, 2015, pp. 19-21. doi: 10.11648/j.ajmp.20150401.14

\begin{abstract}
It is well known that the quasi-exact solvable eigenvalues of the Schrödinger equation with potential $V(x)=-$ $(\xi \cosh 2 \mathrm{x}-i M)^{2}$ is real for PT-invariant non-Hermitian potential in case the parameter $\mathrm{M}$ is odd integer and complex conjugate pairs when $M$ is even integer. In this work the Asymptotic Iteration Method (AIM) were used to solve this problem for M odd and even integer, and for any non-integer $\mathrm{M}$ values.
\end{abstract}

Keywords: Asymptotic Iteration Method, Eigenvalues, Complex Potential, Non- Quasi Exact Solvable (QES)

\section{Introduction}

Recently, there has been a growing interest in the study of non-Hermitian Hamiltonians which appearing in different branches of physics [1, 2, and 3]. The main reason for this is that the energy eigenvalues for a number of complex potentials turned out to be real [4].

Several non-Hermitian PT symmetric potentials with real or complex eigenenergies have been analyzed using a variety techniques, such as perturbation theory[5], semi classical estimations[6], algebraic methods[7], analytical arguments[8], numerical experiments[9], potential algebras [10, 11], supersymmetrization [12] or some generalizations thereof, and quasi-solvability[13].

In this paper we applied the Asymptotic Iteration Method (AIM) to study the complex potential which had been studded by quasi-exactly solvable (QES) technique [14]. They considered the system described by the non-Hermitian PT-invariant potential

$$
V(x)=(\xi \cosh 2 x-i M)^{2}
$$

where the parameter $\zeta$ is real and parameter $M$ has only integer values. They clarify that when the parameter $M$ is odd integer the eigenvalues of the Schrödinger equation is real, and it is complex conjugate pairs when $\mathrm{M}$ is even integer. In the literature the non quasi exact solvable (QES) spectrums for this problem was not revealed yet.

In our work we solved the QES and non QES eigenvalue problem for the complex non-hermitian PT-invariant potential equation (1) by using the Asymptotic Iteration Method (AIM). The results we found for QES case by the AIM technique are in full agreements with the analytical results obtained by Avinash Khare, et. al. [14]. Moreover the Asymptotic Iteration Method (AIM) technique showed very interesting results for the non- quasi exact solvable (QES) case which has not been solved before [15-19].

\section{Method and Calculations}

The Asymptotic Iteration Method (AIM) was used to solve the one dimensional Schrödinger equation in the field of PT invariant complex potential $\mathrm{V}(\mathrm{x})$ equation (1),

$$
H \psi(x)=\left[\frac{p^{2}}{2 m}+V(x)\right] \psi(x)=E_{n} \psi(x)
$$

where $\mathrm{H}$ is a non Hermitian PT invariant Hamiltonian and $(\hbar=2 m=1)$ is in atomic units. The one dimensional wave function $\psi(x)$ of the form

$$
\psi(x)=e^{i \frac{\xi}{2} \cosh 2 x} \phi(x)
$$

was used, $\psi(x) \rightarrow 0$ as $|x| \rightarrow \infty$ on the real axis.

The AIM was applied to this problem without any complicated transformations, and the Schrödinger equation obtained for this potential is of the form, 
$\phi^{\prime \prime}(x)=-4 i a \sinh (x) \cosh (x) \phi^{\prime}(x)$

$-\left(4 i a \cosh ^{2}(x)-2 i a+a^{2}-4 i a M \cosh ^{2}(x)+2 i a M-M^{2}+E\right) \phi(x)$

and could be written as follows

$$
\phi^{\prime \prime}=\lambda_{0}(x) \phi^{\prime}+s_{0}(x) \phi
$$

Where $\lambda_{o}(x)=-4 i a \sinh (x) \cosh (x)$ and

$s_{o}(x)=-\left(4 i a \cosh ^{2}(x)-2 i a+a^{2}-4 i a M \cosh ^{2}(x)+2 i a M-M^{2}+E\right)$

According to the Asymptotic Iteration Method (AIM), we differentiate equation (4) with respect to $\mathrm{x}$, and we get this form,

$$
\phi^{\prime \prime \prime}=\lambda_{1}(x) \phi^{\prime}+s_{1}(x) \phi
$$

Where, $\lambda_{1}(x)=\lambda_{0}^{\prime}+s_{0}+\lambda_{0}^{2}$ and $s_{1}(x)=s_{0}^{\prime}+s_{0} \lambda_{0}$

Thus, for $(\mathrm{n}+1)$ th and $(n+2)^{\text {th }}$ derivatives, $\mathrm{n}=1,2, \ldots \ldots$. , we have:

$$
\phi^{(n+1)}=\lambda_{n-1}(x) \phi^{\prime}+s_{n-1}(x) \phi \text { and } \phi^{(n+2)}=\lambda_{n}(x) \phi^{\prime}+s_{n}(x) \phi
$$

where $s_{n}=s_{n-1}^{\prime}+s_{0} \lambda_{n-1}$ and, $\lambda_{n}=\lambda_{n-1}^{\prime}+s_{n-1}-\lambda_{0} \lambda_{n-1}$

For some suitable large iterations $\mathrm{j}$, one can numerically determine the eigenvalues from the roots of the equation

$$
\delta_{j}=\lambda_{j-1}(x) s_{j}(x)-s_{j-1}(x) \lambda_{j}(x)
$$

\section{Results and Conclusion}

To test our method, the Asymptotic Iteration Method (AIM) was applied to this problem for $\mathrm{M}$ even integer and our results are listed in Table (1) and Table (2). We have obtained exactly the same complex conjugate pairs for the energy eigenvalues that obtained by quasi exactly solvable method (QES). Our results are in full agreements with the results obtained in the literatures. The Asymptotic Iteration Method (AIM) is also applied to the problem when $\mathrm{M}$ is odd integers and the energy eigenvalues were calculated for $\zeta^{2}=0.01$ and the results obtained are listed in Table (3) for $\mathrm{M}=5$ and in Table (4) for $M=7$ and in Table (5) for $M=9$. From these tables one can easily recognized that the Asymptotic Iteration Method (AIM) works smoothly and the results obtained are in good agreements with the quasi exact solvable (QES) method [14]. It is important to mention that the AIM works for any $\mathrm{M}$ odd or even integer, while the QES method face very complicated mathematical formulas for higher $\mathrm{M}$ values.

\section{A non Quasi Exact Solvable (QES) by Asymptotic Iteration Method (AIM)}

We extend our work to treat the non- quasi exact solvable (QES) eigenvalues for the non Hemitian PT- invariant potential Eq. (1) by considering non-integer $\mathrm{M}$ values, which is not available in the quasi exactly solvable method due to the very complicated formulas which appear when $M$ is becoming non-integer. The results obtained in this case were listed in Table (6). The Asymptotic Iteration Method (AIM) showed that the eigenvalues has imaginary parts, and this imaginary part is very small and approaches zero as the noninteger $\mathrm{M}$ values are getting bigger.

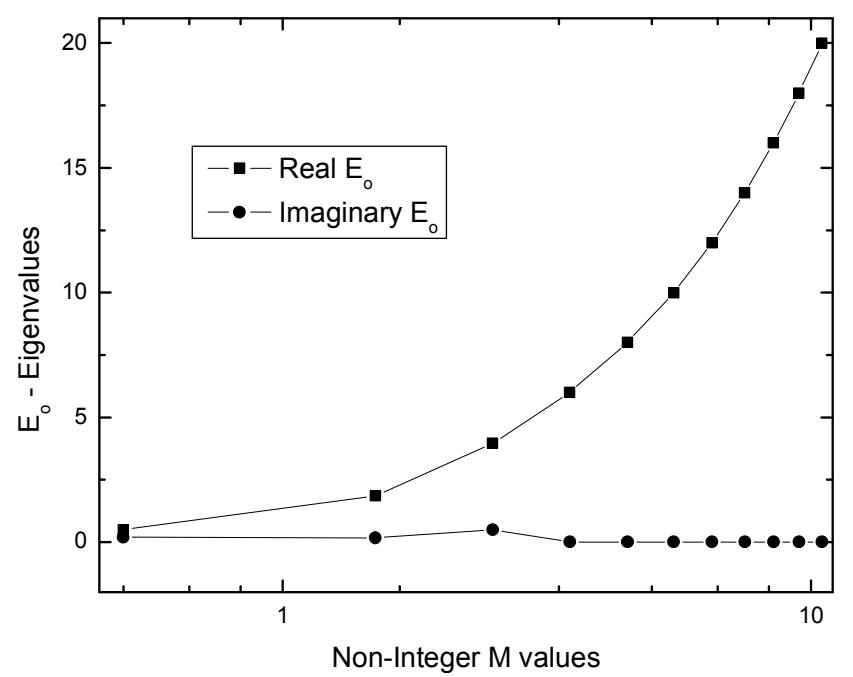

Figure (1). The relation between the real and imaginary part of the eigenvalues versus the non-integer $M$ values.

Figure (1) shows the relation between the real and imaginary part of the eigenvalues versus the non-integer $M$ values. It is important to note that the imaginary parts of the eigenvalues are small enough to be neglected. We conclude that the eigenvalues for non-integer $\mathrm{M}$ values were becoming real, just as in the case when $\mathrm{M}$ is an odd integer. These results are considered to be valuable because they reveal all aspects of this problem which are unable to solve by exact technique.

Table (1). Comparison of the eigenvalues obtained by QES with AIM for $M$ even integer

\begin{tabular}{lll}
\hline \multicolumn{2}{l}{ Energy levels for $\mathbf{M}=\mathbf{2}, \zeta^{2}=0.01$} \\
\hline \multicolumn{2}{l}{ QES } & AIM \\
\hline$E_{ \pm}^{0}$ & $2.99000000 \pm 0.20000000000 i$ & $2.99000000 \pm 0.20000000000 i$ \\
\hline
\end{tabular}

Table (2). Comparison of the eigenvalues obtained by QES with AIM for $M$ even integer

\begin{tabular}{lll}
\hline \multicolumn{2}{l}{ Energy levels for $\mathbf{M}=\mathbf{4}, \zeta^{2}=0.01$} \\
\hline & QES & AIM \\
\hline$E_{ \pm}^{0}$ & $7.004990401 \pm 0.0007523395 i$ & $7.004990401 \pm 0.0007523395 i$ \\
$E_{ \pm}^{1}$ & $14.97500960 \pm 0.4007523395 i$ & $14.97500960 \pm 0.4007523395 i$ \\
\hline
\end{tabular}

Table (3). Comparison of the eigenvalues obtained by QES with AIM for $M$ odd integers

\begin{tabular}{lll}
\hline \multicolumn{2}{l}{ Energy levels for $\mathbf{M}=\mathbf{5}, \zeta^{2}=0.01$} & AIM \\
\hline$E_{0}$ & QES & 9.003314792 \\
$E_{1}$ & 9.00331480 & 9.003348181 \\
$E_{2}$ & 9.00334818 & 20.97665182 \\
$E_{3}$ & 20.97665182 & 21.10018724 \\
$E_{4}$ & 21.10018724 & 24.86649797 \\
\hline
\end{tabular}


Table (4). Comparison of the eigenvalues obtained by QES with AIM for $M$ odd integers

\begin{tabular}{lll}
\hline \multicolumn{2}{l}{ Energy levels for $\mathbf{M}=\mathbf{7}, \zeta^{2}=0.01$} \\
\hline & QES & AIM \\
\hline$E_{o}$ & 13.00199970 & 13.00199970 \\
$E_{1}$ & 13.00199971 & 13.00199971 \\
$E_{2}$ & 33.01123852 & 33.01123852 \\
$E_{3}$ & 33.01140573 & 33.01140573 \\
$E_{4}$ & 44.95659457 & 44.95659457 \\
$E_{5}$ & 45.21134926 & 45.21134926 \\
$E_{6}$ & 48.73541251 & 48.73541251 \\
\hline
\end{tabular}

Table (5). Comparison of the eigenvalues obtained by QES with AIM for M odd integers

\begin{tabular}{lll}
\hline \multicolumn{2}{l}{ Energy levels for $\mathbf{M}=\mathbf{9}, \zeta^{2}=0.01$} \\
\hline & $\mathbf{Q E S}$ & AIM \\
\hline $\mathrm{E}_{\mathrm{o}}$ & 17.00096869 & 17.00142845 \\
$\mathrm{E}_{1}$ & 17.00153729 & 17.00142847 \\
$\mathrm{E}_{2}$ & 45.00607980 & 45.00656978 \\
$\mathrm{E}_{3}$ & 45.00751350 & 45.00656989 \\
$\mathrm{E}_{4}$ & 65.02149678 & 65.02171309 \\
$\mathrm{E}_{5}$ & 65.02298207 & 65.02221570 \\
$\mathrm{E}_{6}$ & 76.92940084 & 76.92978572 \\
$\mathrm{E}_{7}$ & 77.37417833 & 77.37448084 \\
$\mathrm{E}_{8}$ & 80.5458427 & 80.54580802 \\
\hline
\end{tabular}

Table (6). The real and imaginary eigenvalues for non-integer $M$ values computed by (AIM)

\begin{tabular}{lll}
\hline $\mathbf{M}$ & Real part of $\mathbf{E}_{\mathbf{0}}$ & Imaginary Part of $\mathbf{E}_{\mathbf{o}}$ \\
\hline 0.5 & 0.486299 & 0.197849 \\
1.5 & 1.865428 & 0.163822 \\
2.5 & 3.963098 & 0.48799 \\
3.5 & 6.003646 & 0.002973 \\
4.5 & 8.002855 & 0.000083 \\
5.5 & 10.002855 & $0.153127 \times 10^{-5}$ \\
6.5 & 12.002221 & $0.207869 \times 10^{-7}$ \\
7.5 & 14.001818 & $0.223279 \times 10^{-9}$ \\
8.5 & 16.001538 & $0.197518 \times 10^{-11}$ \\
9.5 & 18.001333 & $0.170342 \times 10^{-10}$ \\
10.5 & 20.001176 & $0.836227 \times 10^{-8}$ \\
\hline
\end{tabular}

\section{References}

[1] Carl M. Bender, Stefan Boettcher, Phys. Rev. Lett. 80, 5243 5246 (1998).
[2] Carl M. Bender, Gerald V.Duune, Peter N.Meisnnger, Physics Letters A, Volume 252, Issue 5, page 272- 276 (1 March 1999).

[3] C. Quesen, S. Mallik, B. Bagchi, Int.J.Mod.Phys. A16 2859$2872(2001)$.

[4] S. Meyur, S. Debnath, Bulg. J. Phys. 36 (77- 87), (2009).

[5] Qing-Hai Wang, Pramana Journal of physics, V 37, No .2 (315-322), (2009).

[6] Brice Camus, Journal of Differential Equations, Volume 226, Issue 1, 295- 322 (2006).

[7] M. E. Kellman, Annual Review of Physical Chemistry, vol. 46, pp. 354- 421, (1995).

[8] F. Cannata, G. Junker, and J. Trost, Phys. Lett A 246, 129 (1998).

[9] Tanwa Arpornthip, Carl M. Bender, Pramana Journal of physics, V 37, No. 2 (259- 267), (2009).

[10] G. L'evan, F. Cannata, and A. Ventura, J. Phys. A: Math. Gen. 34,839 (2001).

[11] A. D. Alhaidari, J. Phys. A: Math. Theor. 40, 6305 (2007).

[12] Bijan Bagchi et al, Int. J. Mod. Phys. A 20, 7107 (2005).

[13] C. M. Bender and S. Boettcher, J. Phys. A: Math. Gen. 31, L 273 (1998).

[14] A Khare and B P Mandal, Phys. Lett. A272, 53 (2000).

[15] H. Ciftci, R. L. Hall, and N. Saad, J. Phys. A: Math. Gen. 36, 11807 (2003).

[16] F. M. Fern'andez, J. Phys. A: Math. Gen. 37, 6173 (2004).

[17] A. J. Sous, J. Mod. Phys. Lett. A 21, 1675 (2006).

[18] A. J. Sous and M. I. EL-Kawni, Int. J. Mod. Phys. A 24, 4169 (2009).

[19] T. Barakat 2006 J. Phys. A: Math. Gen. 39 823, Issue 4 (27 January 2006). 